\title{
Exact solutions for universal holonomic quantum gates
}

\author{
V. Karimipoun* and N. Majd \\ Department of Physics, Sharif University of Technology, P.O.Box 11365-9161, Tehran, Iran
}

(Dated: October 27, 2018)

\begin{abstract}
We show how one can implement any local quantum gate on specific qubits in an array of qubits by carrying adiabatically a Hamiltonian around a closed loop. We find the exact form of the loop and the Hamiltonian for implementing general one and two qubits gates. Our method is analytical and is not based on numerical search in the space of all loops.
\end{abstract}

*Electronic address: vahid@sharif.edu

${ }^{\dagger}$ Electronic address: naymajd@mehr.sharif.edu 


\section{INTRODUCTION}

Since the suggestion of Zanardi and Rasetti in [1] on holonomic quantum computation and its further development in [2, 3, 4, 5, 6] concrete realization of holonomic quantum gates has attracted a lot of attention. The attraction comes mainly from the fact that this type of quantum computation may be inherently stable against some local errors. The basic idea of 1] is as follows. One takes an $n$ dimensional Hamiltonian with a degenerate $k$ dimensional eigenspace

$$
H(t)|i(t)\rangle=\epsilon(t)|i(t)\rangle
$$

where $\{|i(t)\rangle, \quad i=1 \quad k\}$ are the instantenous degenerate eigenstates of the Hamiltonian. Carrying this hamiltonian adiabatically around a loop in the parameter space, induces a generalization of the Berry phase in the form of a unitary operator in this subspace. This unitary operator is given by

$$
U=P e^{-\oint_{C} A}
$$

where $P$ means path-ordered exponential, $A$ is the connection one form

$$
\langle i(t)|A| j(t)\rangle:=\langle i(t)|d| j(t)\rangle, \quad i, j=1 \text { to } k
$$

and $C$ is the loop around which the Hamiltonian is moved in the parameter space. Here we are assuming that the basis vectors of the Hilbert space have been arranged so that the first $k$ states span the degenerate eigenspace of the Hamiltonian.

Of particular interest to us is a scenario first proposed from a geometrical point of view in 7]. The physical description of the idea of [7] is as follows: To make the connection one form time independent, one takes a family of iso-spectral Hamiltonians on an $n$ dimensional Hilbert space defined as follows:

$$
H(t)=e^{t X} H_{0} e^{-t X}
$$

where $H_{0}=\epsilon \sum_{i=1}^{k}|i\rangle\langle i| \equiv \epsilon P_{0}$, and $X$ is an anti-hermitian operator acting on the Hilbert space. Thus $P_{0}$ is the projection operator for the $k$ - dimensional eigenspace, and the operator $X$ determines the adjoint action and hence the curve in the parameter space. Note that the curve has been parameterized by the real parameter $t \in[0,1]$ and if one requires that the curve be closed in the parameter space, one should have

$$
P_{0}=e^{X} P_{0} e^{-X}
$$


It is always possible to choose the basis of the Hilbert space so that

$$
P_{0}=\left(\begin{array}{cc}
I_{k \times k} & 0 \\
0 & 0
\end{array}\right) \text {. }
$$

For the Hamiltonian (4) we have:

$$
|i(t)\rangle=e^{t X}|i\rangle
$$

from which we obtain the time independent connection

$$
\langle i(t)|A| j(t)\rangle=\langle i|X| j\rangle, \quad i, j=1 \quad \cdots \quad k .
$$

Note that this equation by no means implies the $A=X$. It only means that the restriction of the operator $X$ to the degenerate subspace is equal to $A$.

Since $X$ is constant, calculation of the path integral is quite simple and gives

$$
U=P e^{-\oint_{C} A}=e^{-A}
$$

Note that the operator $X$ determines the curve in the parameter space completely. However only its projection to the degenerate subspace is determined by the operator $U=e^{-A}$ which we want to implement $\varpi$ holonomically through equation (8), and one is free to choose the rest of this operator to satisfy the constraint (5) which means that the curve should be a closed one. As an explicit example [7], if one wants to implement a one qubit gate $u=e^{-A} \in U(2)$ one should take $k=2$ and can take $n=3$ and $X$ as

$$
X=\left(\begin{array}{cc}
A & w \\
-w^{\dagger} & 0
\end{array}\right),
$$

where $w$ is a two dimensional vector which should be determined by satisfying the constraint (5). In [7] the dimension $n$ is always taken to be $k+1$ and solutions for some one quibt gates (e.g. the Hadamard gate) for which $k=2$ and two qubit gates (e.g. the CNOT gate and the 2 dimensional fourier transform) for which $k=4$ are obtained by extensive numerical search in the space of two and four dimensional vectors respectively.

Our aim in this paper is two-fold. First we provide an exact method (free of numerical calculations) for obtaining solutions for arbitrary unitary gates acting on an arbitrary number of qubits. We obtain in a much simpler method the solutions already obtaind in 7]. Second we address the problem of scalability of this construction. That is, we modify 
the method of [7] so that we can holonomically implement any local quantum gate on an array of qubits by appropriate choices of curves in the parameter space. This will give us full power for doing holonomic computation on a quantum computer in a scalable way.

The structure of the paper is as follows: In section II we present our exact solution for the case when $n=k+1$. Since this is the case already studied by the authors in [7], who have supplied various examples by searching in the space of matrices numerically, we suffice to give only proofs for general gates and refrain from giving examples. In section III we generalize our method and show how to holonomically implement any local quantum gate on an array of qubits by appropriate choices of curves in the parameter space.

We end the paper by a short discussion.

\section{ANALYTICAL SOLUTION}

Let us take an arbitrary anti-hermitian operator $X \in u(n)$. This determines the adjoint orbit via equation (44). Due to equation (8) and the fact that $n=k+1$, the general form of $X$ is as follows:

$$
X=\left(\begin{array}{cc}
A & w \\
-w^{\dagger} & i s
\end{array}\right),
$$

where $s$ is a real number and $i=\sqrt{-1}$. Note that the upper $k$ by $k$ diagonal block of $X$ is dictated by the choice of unitary operator $U=e^{-A}$ that we want to represent holonomically. We have choices only for the vector $w$ and the number $s$. We can use this freedom to solve the constraint (5) which requires that the loop be closed.

In 7] the parameter $s$ has been taken equal to zero. However we will see that a better choice for this parameter exists which will simplify the final results considerably. Our task is now to exponentiate the operator $X$.

Exponentiation of a specific anti-hermitian operator is a straightforward (albeit highly tedius) task which can be done by diagonalization of the operator (since we have to solve cubic equations with general coefficients). Since we want to do this for a completely general operator of the form (11), for which we have no a priori information about the matrix $A$ and the vector $w$, we make the simplifying restriction that $w$ is proportional to an eigenvector 
of $A$, that is

$$
A w=i \lambda w, \quad w^{\dagger} w=\alpha^{2},
$$

where $\lambda$ is a real parameter.

With this assumption we can use a recursive method for calculation of $e^{t X}$ instead of numerical methods.

A simple calculation for the first few powers shows that the general form of $X^{n}$ is

$$
X^{n}=\left(\begin{array}{cc}
A^{n}-b_{n} w w^{\dagger} & c_{n} w \\
-c_{n}^{*} w^{\dagger} & d_{n}
\end{array}\right),
$$

where $a(n), b(n)$ and $c(n)$ are coefficients to be determined. These coefficients satisfy the following initial conditions:

$$
\begin{array}{ll}
b_{0}=0 & b_{1}=0, \\
c_{0}=0 & c_{1}=1, \\
d_{0}=1 & d_{1}=i s .
\end{array}
$$

To obtain these coefficients we use

$$
\begin{aligned}
& X^{n+1}=\left(\begin{array}{cc}
A & w \\
-w^{\dagger} & i s
\end{array}\right)\left(\begin{array}{cc}
A^{n}-b_{n} w w^{\dagger} & c_{n} w \\
-c_{n}^{*} w^{\dagger} & d_{n}
\end{array}\right) \\
& =\left(\begin{array}{cc}
A^{n+1}-b_{n+1} w w^{\dagger} & c_{n+1} w \\
-c_{n+1}^{*} w^{\dagger} & d_{n+1}
\end{array}\right) \text {, }
\end{aligned}
$$

from which we obtain the following recursive system of equations

$$
\begin{aligned}
& b_{n+1}=i \lambda b_{n}+c_{n}^{*}, \\
& c_{n+1}=i \lambda c_{n}+d_{n}, \\
& d_{n+1}=i s d_{n}-\alpha^{2} c_{n} .
\end{aligned}
$$

To solve this system we define the following generating functions

$$
\begin{aligned}
B(t) & :=\sum_{n=0}^{\infty} \frac{b_{n}}{n !} t^{n}, \\
C(t) & :=\sum_{n=0}^{\infty} \frac{c_{n}}{n !} t^{n}, \\
D(t) & :=\sum_{n=0}^{\infty} \frac{d_{n}}{n !} t^{n} .
\end{aligned}
$$


Once we find these generating functions we have the exponential as

$$
e^{t X}=\left(\begin{array}{cc}
e^{t A}-B(t) w w^{\dagger} & C(t) w \\
-C^{*}(t) w^{\dagger} & D(t)
\end{array}\right) .
$$

In terms of generating functions the recursion relations read as follows:

$$
\begin{aligned}
& B^{\prime}(t)=i \lambda B(t)+C^{*}(t), \\
& C^{\prime}(t)=i \lambda C(t)+D(t), \\
& D^{\prime}(t)=i s D(t)-\alpha^{2} C(t) .
\end{aligned}
$$

The second and the third equations can be combined to give

$$
C^{\prime \prime}(t)=i(\lambda+s) C^{\prime}(t)+\left(\lambda s-\alpha^{2}\right) C(t)
$$

the solution of which is

$$
C(t)=\gamma_{1} e^{q_{1} t}+\gamma_{2} e^{q_{2} t}
$$

where

$$
q_{1,2}=\frac{i}{2}\left(\lambda+s \pm \sqrt{(\lambda-s)^{2}+4 \alpha^{2}}\right)=: i\left(\frac{\lambda+s}{2} \pm \nu\right)
$$

where we have defined $\nu:=\frac{1}{2} \sqrt{(\lambda-s)^{2}+4 \alpha^{2}}$ and $\gamma_{1,2}$ are constants to be determined from initial conditions.

In view of the initial conditions

$$
c_{0} \equiv C(0)=0 \quad \text { and } \quad c_{1} \equiv C^{\prime}(0)=1,
$$

we find the final form of the generating function

$$
C(t)=\frac{e^{q_{1} t}-e^{q_{2} t}}{q_{1}-q_{2}}=e^{i \frac{(\lambda+s) t}{2}} \frac{\sin \nu t}{\nu} .
$$

From this solution we find that a simplifying choice for the parameter $s$ is to take $s=-\lambda$ for which we will have:

$$
C(t)=\frac{\sin \nu t}{\nu}
$$

where now

$$
\nu=\sqrt{\lambda^{2}+\alpha^{2}}
$$

. Equation (19) will then give

$$
D(t)=C^{\prime}(t)-i \lambda C(t)=\cos \nu t-i \frac{\lambda}{\nu} \sin \nu t .
$$


Finally we solve the first equation of (19) by rewriting it as

$$
\frac{d}{d t}\left(e^{-i \lambda t} B(t)\right)=e^{-i \lambda t} C^{*}(t)
$$

the solution of which is

$$
\begin{aligned}
B(t) & =\int_{0}^{t} e^{i \lambda(t-\tau)} C^{*}(\tau) d \tau \\
& =\frac{1}{\left(\nu^{2}-\lambda^{2}\right)}\left(e^{i \lambda t}-\cos \nu t-i \frac{\lambda}{\nu} \sin \nu t\right),
\end{aligned}
$$

where we have used the initial condition $b_{0} \equiv B(0)=0$.

What we now require is that the constraint (5) be satisfied. This constraint is satisfied when the operator $e^{X}$ becomes block-diagonal, since in this case $\left[e^{X}, P_{0}\right]=0$. Due to equation (18) this requires that we set $C(1)=0$ which in view of (24) demands that we fix $\nu$ as $\nu=\pi n$ or in view of equation (26),$\alpha^{2}=(n \pi)^{2}-\lambda^{2}$, where $n$ is an integer.

Thus the recepie is as follows: for any given operator $U=e^{-A}$, we choose an eigenvector $v$ of $A$, we form the matrix $X$ as in (11) with $w=\alpha v$ where $\alpha$ is given as $\alpha^{2}=(n \pi)^{2}-\lambda^{2}$. Thus for any operator in a $k$ dimensional space there are a number of $k$ closed curves, each corresponding to one of the eigenvectors of $A$. In view of equation (24), for each fixed $n$ the Hamiltonian $H(t)$ turns back to its original form $H_{0}$ exactly $n$ times during the period from $t=0$ to $t=1$. The optimal choice for $n$ is thus $n=1$.

\section{GENERALIZATION AND PHYSICAL REALIZATION}

Let us summarize our findings up to now. If we want to implement a unitary operator (a quantum gate) on a $k$ dimensional Hilbert space, we take a system whose Hilbert space is $k+1$ dimensional. On this larger Hilbert space, we take a Hamiltonian $H_{0}=P_{0}$, where $P_{0}$ is the projector operator on the smaller $k$ dimensional system and then adiabatically transform this initial Hamiltonian by the adjoint action $H(t)=e^{t X} P_{0} e^{-t X}$, where $X$ is a vector in the lie algebra $\in u(k+1)$. For any given unitary operator $U \in U(k)$, we have a suitable vector $X$, such that the holonomy operator which is adiabatically induced on the degenerate $k$ dimensional Hilbert space coincides with that operator, that is $e^{-\oint_{C} A}=U$, where $\langle i(t)|A| j(t)\rangle=\langle i(t)|d| j(t)\rangle=\langle i|X| j\rangle, \quad 1 \leq i, j \leq k$.

Actually for any given operator $U$, we have $k$ generally different solutions $X$, since we have $k$ 
different choices for the vector $w$ to insert in (11), each proportional to a different eigenvector of the operator $A$. This is an important point which we will come to later in this section. In fact we will see that it is best to combine the separate solutions into one single solution. The reason is that when it comes to physical realization of the technique in section (III), the problem of dimension becomes an annoying obstacle, since if we want to implement a single qubit gate, we require a three dimensional Hilbert space and if we want to implement a two-qubit gate, we need a five dimensional Hilbert space and so on. Generally we do not have such a control over the dimensions of Hilbert spaces in most experimental scenarios for quantum computation. In most of these scenarios we have a number $n$ of two dimensional systems (e.g. spins, ions, etc.) which play the role of qubits which when put together make a Hilbert space of dimension $2^{n}$. When one enlarges this system by adding a quibt (an ancilla qubit) the dimension of the Hilbert space doubles to $2^{n+1}$ instead of getting increased by one.

The most ideal situation is to have a main array of qubits numbered from 1 to $n$ together with an ancilla qubit which we number as $n+1$. This ancilla qubit is then used for implementing holonomically a set of universal gates (one qubit and two qubit gates) on any qubit or pair of qubits in the main array of qubits.

In order to realize this ideal situation we modify our method as follows. We let the dimension of the larger Hilbert space to be twice the dimension of the degenerate subspace. For simplicity of presentation we first consider the example where we have a single qubit as our main system and a single ancilla qubit. Instead of (11) we now take $X$ as follows:

$$
X=\left(\begin{array}{cc}
A & \left(w_{1}, w_{2}\right) \\
-\left(\begin{array}{c}
w_{1}^{\dagger} \\
w_{2}^{\dagger}
\end{array}\right) & \left(\begin{array}{cc}
-i \lambda_{1} & 0 \\
0 & -i \lambda_{2}
\end{array}\right)
\end{array}\right)
$$

where $w_{1}=\alpha_{1} v_{1}$ and $w_{2}=\alpha_{2} v_{2}$ are proportional to the two eigenvectors of the operator $A$ corresponding to eigenvalues $\lambda_{1}$ and $\lambda_{2}$ respectively. In this way we incorporate the two separate solutions into one solution for the sake of scalability of holonomic computation. We can easily repeat all the calculations in section (III). For example we will have

$$
X^{n}=\left(\begin{array}{cc}
A^{n}-b_{1, n} w_{1} w_{1}^{\dagger}-b_{2, n} w_{2} w_{2}^{\dagger} & \left(c_{1, n} w_{1}, c_{2, n} w_{2}\right) \\
-\left(\begin{array}{cc}
c_{1, n}^{*} w_{1}^{\dagger} \\
c_{2, n}^{*} w_{2}^{\dagger}
\end{array}\right) & \left(\begin{array}{cc}
d_{1, n} & 0 \\
0 & d_{2, n}
\end{array}\right)
\end{array}\right)
$$


where the functions $b_{k, n}, c_{k, n}$ and $d_{k, n}$ for $k=1,2$ are found to satisfy the following recursion relations:

$$
\begin{aligned}
b_{k, n+1} & =i \lambda_{k} b_{k, n}+c_{k, n}^{*}, \\
c_{k, n+1} & =i \lambda_{k} c_{k, n}+d_{k, n}, \\
d_{k, n+1} & =-i \lambda_{k} d_{k, n}-\alpha_{k}^{2} c_{k, n}^{*} .
\end{aligned}
$$

This shows that the recursion relations for various indices $k$ are decoupled and thus the solutions for the generating functions found in section (III) can be directly carried over to here. For example we have for each $k$, that

$$
C_{k}(t):=\frac{\sin \nu_{k} t}{\nu_{k}}
$$

where $\nu_{k}=\sqrt{\lambda^{2}+\alpha_{k}^{2}}$. This tells us that the matrix $e^{X}$ will be block diagonal if we choose $\nu_{k}=n_{k} \pi$ or optimally if $\nu_{k}=\pi$.

A more concise and instructive way of doing the above calculation is to rewrite $\left(w_{1}, w_{2}, \cdots w_{n}\right)$ as

$$
\left(w_{1}, w_{2}, \cdots w_{n}\right)=\left(\alpha_{1} v_{1}, \alpha_{2} v_{2}, \cdots \alpha_{n} v_{n}\right)=\Omega D
$$

where $\Omega$ is the unitary matrix which diagonalizes $A$ and $D=\operatorname{diagonal}\left(\alpha_{1}, \alpha_{2}, \cdots \alpha_{n}\right)$. Then we can write $X$ in the form

$$
X=\left(\begin{array}{cc}
A & \Omega D \\
-D \Omega^{\dagger} & -i \Lambda
\end{array}\right)=\left(\begin{array}{cc}
\Omega & 0 \\
0 & I
\end{array}\right)\left(\begin{array}{cc}
i \Lambda & D \\
-D & -i \Lambda
\end{array}\right)\left(\begin{array}{cc}
\Omega^{\dagger} & 0 \\
0 & I
\end{array}\right) .
$$

In this form the matrix $X$ is easily exponentiated to give

$$
e^{t X}=\left(\begin{array}{cc}
\Omega & 0 \\
0 & I
\end{array}\right) e^{t X_{0}}\left(\begin{array}{cc}
\Omega^{\dagger} & 0 \\
0 & I
\end{array}\right)
$$

where the matrix $X_{0}$ has been defined as

$$
X_{0}:=\left(\begin{array}{cc}
i \Lambda & D \\
-D & -i \Lambda
\end{array}\right)
$$

It is clear that

$$
\begin{aligned}
& X_{0}=i\left(\sigma_{z} \otimes \Lambda+\sigma_{y} \otimes D\right) \\
& X_{0}^{2}=-I \otimes\left(\Lambda^{2}+D^{2}\right) .
\end{aligned}
$$


Note that both $\Lambda$ and $D$ are diagonal matrices. This then leads to the following result

$$
e^{X_{0} t}=I \otimes \cos \nu t+i\left(\sigma_{z} \otimes \Lambda+\sigma_{y} \otimes D\right)\left(I \otimes \frac{\sin \nu t}{\nu}\right),
$$

where $\nu:=\sqrt{\Lambda^{2}+D^{2}}$ is a diagonal matrix which generalizes and replaces the simple factors $\nu=\sqrt{\lambda^{2}+\alpha^{2}}$ of section (III). The condition (5) is now satisfied if we choose

$$
\nu_{k}=\sqrt{\lambda_{k}^{2}+\alpha_{k}^{2}}=n_{k} \pi \quad \forall k .
$$

If we take all these factors equal to $\pi$ which is the optimal choice for each factor, the matrix $\nu$ will equal $\pi I$. This brings about further simplifications as we will see.

From (36) and (39), we find that

$$
e^{t X}=\left(\begin{array}{cc}
\cos \pi t+\frac{A}{\pi} \sin \pi t & \frac{\Omega D}{\pi} \sin \pi t \\
-\frac{D \Omega^{\dagger}}{\pi} \sin \pi t & \cos \pi t-i \frac{\Lambda}{\pi} \sin \pi t
\end{array}\right) .
$$

Now that we have obtained the general form of the operator $X$, for any unitary operator, let us see what is its form for a one qubit gate $u:=e^{a}$ acting on one of the qubits, say qubit $k$, in the main array. Then we will have

$$
\begin{array}{ll}
A=I^{\otimes k-1} \otimes a \otimes I^{\otimes^{n-k}}, & \Omega=I^{\otimes k-1} \otimes \varpi \otimes I^{\otimes^{n-k}}, \\
\Lambda=I^{\otimes k-1} \otimes \lambda \otimes I^{\otimes^{n-k}}, & D=I^{\otimes k-1} \otimes d \otimes I^{\otimes^{n-k}},
\end{array}
$$

where $\varpi$ is the matrix which diagonalizes $a, \lambda$ is the diagonalization of $a$, and $d$ is the diagonal matrix $d=\operatorname{diag}\left(\alpha_{1}, \alpha_{2}\right)$, where $\pi=\sqrt{\lambda_{k}^{2}+\alpha_{k}^{2}}$. Thus we see that for a local one qubit gate $u_{k}=I^{\otimes k-1} \otimes u \otimes I^{\otimes n-k}$ which acts trivially on all other qubits except the $k$-th qubit, our matrix $X$ will also be a local matrix

$$
X_{k}:=I^{\otimes k-1} \otimes x \otimes I^{\otimes n-k},
$$

is a matrix acting on the $k$-th qubit and the ancilla and $x$ is a four by four matrix of the form (35), that is $x=\left(\begin{array}{cc}a & \varpi d \\ -d \varpi^{\dagger} & -i \lambda\end{array}\right)$.

By the same analysis, we find that a local two qubit gate can also be implemented holonomically, by a local choice of $X$ acting only on the two qubits and the ancilla. The general form of the matrix $X$ is always as given in (35).

For $H_{0}$ we can assume the following form $H_{0}=\frac{1}{2}\left(I+\sigma_{z}\right) \otimes I$, where the first factor acts on the ancilla and the second factor acts on all the other qubits. Thus the Hamiltonian 
$H_{0}$ describes an array of qubits in which only the ancilla qubit is in a magnetic field in the $z$ direction. The eigenstates of $H_{0}$ are $|0\rangle \otimes\left|e_{k}\right\rangle, k=1 \quad$ to $2^{n}$ with energy $E=1$ and $|1\rangle \otimes\left|e_{k}\right\rangle, k=1$ to $2^{n}$ with energy $E=0$, where $\left|e_{k}\right\rangle$ are the basis vectors of the main array of qubits. Thus the Hamiltonian is the projection operator on the main array. According to (7), one now moves arround the orthonormal frame $\left\{|0\rangle \otimes\left|e_{k}\right\rangle\right\}$, by the action

of the operator $e^{t X}$ which means that the Hermitian operator $i X$ acts like a Hamiltonian in the fixed frame. After one cycle of revolution from $t=0$ to $t=1$, the desired operator corresponding to the choice of $X$ has been implemented holonomically on the main array. For a one qubit gate, the operator $x$ can be written in terms of Pauli matrices in the form

$$
x=\frac{1}{2}\left(I+\sigma_{z}\right) \otimes a-\frac{i}{2}\left(I-\sigma_{z}\right) \otimes \lambda+\sigma_{+} \otimes \varpi d-\sigma_{-} \otimes d \varpi^{\dagger} .
$$

Here the first factor of the tensor product acts on the ancilla and the second factor acts on a local qubit. As a concrete example if we take $u=\sigma_{z}$, for which $a=\frac{i \pi}{2}\left(I-\sigma_{z}\right)$ and $\varpi=I$, and follow the above construction, after straightforward calculations we find the following form of the operator $x$ :

$$
x=\frac{i \pi}{2}\left[\left(\sigma_{z}-\sigma_{y}\right) \otimes I+\left(\sigma_{y}-I\right) \otimes \sigma_{z}\right]
$$

\section{DISCUSSION}

We have found exact solutions for the holonomic implementation of local quantum gates on an arbitrary array of qubits plus an ancilla. A family of isospectral Hamiltonians all with the same spectrum of the projection operator on the main array (without the ancilla) is constructed by the choice of a suitable curve in the form of $H(t)=e^{t X} H_{0} e^{-t X}$, defined by an operator in the full Hilbert space of the array and the ancilla. For every single qubit gate acting on the $k$-th qubit, there is a local operator $X_{k}$ acting nontrivially only on the ancilla and the qubit in question, which implements that gate on the $k$-th qubit holonomically. This is also the case for two qubit gates.

Thus by imbedding the appropriate operators for a suitable set of one and two qubit gates which comprize a universal set of quantum gates, in various places in the tensor product of the array, one can enact holonomically any set of gates on any subset of qubits.

This of course leads us to the actual physical implementation of such a method in a concrete physical realization of quantum computers. We leave this problem for future research. 
Acknowledgement We hereby thank A. Sadrolashrafi and A. T. Rezakhani for valuable discussions. After submitting this paper we were informed by the authors of [7] that they have announced a similar solution (without proof) in their revised paper.

[1] Zanardi P. and Rasetti M., Phys. Lett. A. 264, 94(1999).

[2] Ellinas D. and Pachos J., Phys. Rev. A. 64, 022310(2001).

[3] Pachos J., Zanardi P. Rasetti M., Phys. Rev. A. 61, 010305(R)(1999).

[4] Pachos J., and Zanardi P., Int. J. Mod. Phys. 15, 1257(2001).

[5] Pachos J. and Chountasis S., Phys. Rev. A. 62, 052318(2000).

[6] Fujii K. , Rep. Math. Phys. 48, 75 (2001).

[7] Tanimura S., Hayashi D., and Nakahara M., Exact solutions of holonomic quantum computation, quant-phys/0312079 v2. Physics Letters A, Vol 325 No 3-4 pp 199-205. 